\title{
Glottopol
}

Revue de sociolinguistique en ligne

$35 \mid 2021$

La langue à l'école, de l'institution à la classe : quelles conceptions, quelles normes, pour quels usages?

\section{Shana Poplack, 2018, Borrowing: Loanwords in the speech community and in the grammar}

New York: Oxford University Press; xxi, 246 p.

\section{Robert Fournier}

\section{OpenEdition}

Journals

Édition électronique

URL : https://journals.openedition.org/glottopol/311

DOI : $10.4000 /$ glottopol.311

ISSN : 1769-7425

Éditeur

Presses universitaires de Rouen et du Havre

Référence électronique

Robert Fournier, «Shana Poplack, 2018, Borrowing: Loanwords in the speech community and in the grammar », Glottopol [En ligne], 35 | 2021, mis en ligne le 01 janvier 2021, consulté le 02 octobre 2021 URL : http://journals.openedition.org/glottopol/311 ; DOI : https://doi.org/10.4000/glottopol.311

Glottopol 


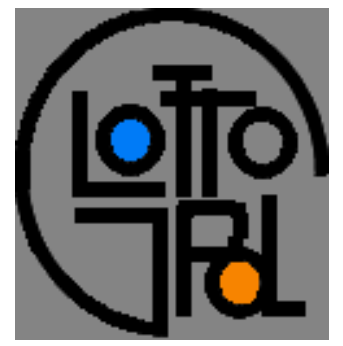

\section{GLOTTOPOL}

Revue de sociolinguistique en ligne $\mathrm{n}^{\circ} 35$ - janvier 2021

\section{La langue à l'école, de l'institution à la classe : quelles conceptions, quelles normes, pour quels usages?}

\section{SOMMAIRE}

Catherine Delarue-Breton et Élisabeth Bautier : Introduction

Catherine Delarue-Breton : Des conceptions scolaires de la langue en tension : l'exemple du programme de 2015 pour l'école primaire et le collège

Véronique Miguel-Addisu : "Sport de riche je l'ai changé en sport élitiste » : plurilinguisme et variation dans la langue de l'école du point de vue des élèves

Claire Colombel-Teuira, Véronique Fillol : Variation et approche polynomique : pour une conception plurielle de la langue à l'école calédonienne

Daphné Bloch : Pratiques langagières, situations pédagogiques et construction d'inégalités d'apprentissage à Madagascar

Samatar Abdallah Doualeh : Le français langue de l'école djiboutienne ou l'hégémonie de la norme exogène

Élise Vinel, Élisabeth Bautier : Des discours des élèves sur l'orthographe aux pratiques des enseignants, analyse d'entretiens métagraphiques

Thierry Pagnier, Belinda Lavieu-Gwozdz: Regards sur le discours scolaire: saisir des conceptions de la langue et de son enseignement

Jacques Crinon, Georges Ferone, Hélène Font : Les enseignants et l'orthographe, une enquête au cycle 3

Christel Troncy : Les enseignants, la norme scolaire et la pluralité langagière dans deux écoles immersives à programme français en Californie. Dynamiques des attitudes et des pratiques lors d'une recherche collaborative

Erwan Le Pipec: L'école, planche de salut du breton?

Argia Olçomendy : La langue basque dans les instructions officielles (1976-2019)

\section{Compte rendu de lecture}

Par Katrin Pfadenhauer: Ursula Reutner (Ed.), 2017, Manuel des francophonies, Berlin/Boston, de Gruyter, 745 p.

Par Robert Fournier: Shana Poplack, 2018, Borrowing: Loanwords in the speech community and in the grammar. New York: Oxford University Press ; xxi, $246 \mathrm{p}$.

Par Marie-Jeanne Verny: Catherine Adam, 2020, Bilinguisme scolaire. Familles, écoles, identités en Bretagne, éd. Peter Lang, coll. «Langue, multilinguisme et changement social », Berlin. 


\section{COMPTE RENDU}

Catherine Adam, 2020, Bilinguisme scolaire. Familles, écoles, identités en Bretagne, éd. Peter Lang, coll. « Langue, multilinguisme et changement social», Berlin.

\section{par Marie-Jeanne Verny \\ UR LLACS - Langues, Littérature, arts et cultures des Suds- Université Paul-Valéry Montpellier 3}

Cet ouvrage, comme l'indique la quatrième de couverture, propose une réflexion sur le bilinguisme scolaire à partir du cas de la scolarisation en classes primaires bilingues en Bretagne. L'objectif de la recherche est d'étudier les représentations que se font les parents d'élèves et, dans une moindre mesure, les enfants concernés, quant au choix de ce type de scolarisation, à partir d'une vaste enquête sous les deux formes d'un questionnaire et d'entretiens semi-directifs. Le terrain en a été soigneusement défini : 3 points différents de la Bretagne offrant tous les trois les trois filières bilingues bretonnes :

- Enseignement bilingue à parité horaire en école publique

- Enseignement bilingue à parité horaire en école privée confessionnelle

- Enseignement immersif en filière associative Diwan de statut privé.

Le traitement de l'enquête est précédé par un long exposé méthodologique sur le «cadre théorique » et les "réflexions épistémologiques » (chapitre 1) qui s'ajoute à 30 pages d'introduction, théorique elle aussi. Si on salue l'honnêteté intellectuelle de l'auteure qui n'a entrepris son étude de corpus qu'une fois lestée d'un solide bagage théorique, on regrette que ces 90 pages n'aient pas été condensées : on se serait contenté d'une bonne bibliographie, bien organisée, qui figure par ailleurs dans l'ouvrage. Et on aurait préféré, dans ce chapitre 1, une présentation synthétique des apports théoriques les plus convaincants.

Plus intéressante, en revanche, se trouve être la présentation précise des spécificités bretonnes quant à la contextualisation sociolinguistique de la langue (chapitre 2) ou aux trois systèmes scolaires (chapitre 3), situation très spécifique, qui n'a pas son équivalent en occitan, et que l'on pourrait comparer au terrain basque.

Le corpus recueilli est très abondant. L'analyse des réponses au questionnaire et des propos tenus, illustrée de schémas, met en lumière un continuum dans les motivations des parents réparties en 8 groupes. Les conclusions sur la typologie de ces motivations des parents et de leurs reflets (étudiés avec précision et dans leur complexité) dans les propos des enfants sont intéressantes et on adhère sans peine aux catégories définies, de $\mathrm{A}$ à $\mathrm{G}$ en allant des stratégies 
d'utilité et des choix pragmatiques (A) à un engagement plus militant $(\mathrm{G})$ vers la transmission d'une langue et d'une culture.

Mais on regrette des lacunes dans les questions posées :

- on ne demande pas aux parents quel système ils ont choisi, ni les raisons de leur choix, alors que les trois filières d'enseignement bilingue du breton ont été soigneusement définies au début de l'ouvrage, et alors que l'auteure a choisi ses trois lieux d'investigation en fonction de la présence, dans chacun d'eux, de ces trois types d'enseignement.

- on ne demande pas aux parents s'ils appartiennent à une association ou non, alors que l'influence de ces associations est clairement étudiée par ailleurs.

Or il aurait été intéressant d'établir une corrélation entre les «types » de parents (de A à G) et leur choix de tel ou tel système ainsi que leur appartenance ou non à une association de type militant.

On peut enfin, de façon plus générale, regretter que ce travail considérable, qui témoigne d'une grande honnêteté intellectuelle et d'un grand souci du détail (au détriment, parfois, de la vision synthétique) soit desservi par un certain laisser-aller éditorial. Visiblement, la relecture professionnelle a été insuffisante et a laissé échapper beaucoup de redites, de maladresses d'expression et de négligences de langue.

Il reste que ce livre contient d'utiles synthèses sur la situation du breton en diachronie et en synchronie, illustrées de schémas fort utiles et que l'enquête de terrain apporte d'utiles éclairages sur les motivations des parents. 


\section{GLOTTOPOL}

Revue de sociolinguistique en ligne

Comité de rédaction : Michaël Abecassis (University of Oxford), Salih Akin (Université de Rouen Normandie), Sophie Babault (Université de Lille), Aude Bretegnier (Université du Mans), Claude Caitucoli, Véronique Castellotti (Université de Tours), Régine DelamotteLegrand (Université de Rouen Normandie), Alexandre Duchêne (Université de Fribourg), Valentin Feussi (Université d'Angers), Robert Fournier (Carleton University, Ottawa), Stéphanie Galligani (Université Grenoble Alpes), Médéric Gasquet-Cyrus (Université AixMarseille), Emmanuelle Huver (Université de Tours), Normand Labrie (Université de Toronto), Foued Laroussi (Université de Rouen Normandie), Benoit Leblanc (Université du Québec à Trois-Rivières), Mylène Lebon-Eyquem (Université de la Réunion), Fabienne Leconte (Université de Rouen Normandie), Gudrun Ledegen (Université de Rennes), Danièle Moore (Simon Fraser University, Vancouver), Clara Mortamet (Université Jean Monnet, Saint Etienne), Alioune Ndao (Université Cheik Anta Diop, Dakar), Isabelle Pierozak (Université de Tours), Cécile Van den Avenne (Université Sorbonne Nouvelle, Paris 3).

Rédactrice en chef : Clara Mortamet.

Directrice de publication : Fabienne Leconte.

Comité scientifique: Michelle Auzanneau (Université de Paris), Margaret Bento (Université de Paris), Jacqueline Billiez (Université de Grenobles Alpes), Philippe Blanchet (Université de Rennes), Jean-Michel Eloy (Université d'Amiens), Françoise Gadet (Université Paris Nanterre), Monica Heller (Université de Moncton), Caroline Juillard (Université de Paris), Jean-Marie Klinkenberg (Université de Liège), Marinette Matthey (Université Grenoble Alpes), Marie-Louise Moreau (Université de Mons-Hainault), Robert Nicolaï (Université Côte d'Azur), Didier de Robillard (Université de Tours), Valérie Spaëth (Université Sorbonne nouvelle), Claude Truchot (Université de Strasbourg), Daniel Véronique (Université AixMarseille).

\section{Comité de lecture pour ce numéro :}

Nathalie Auger, Michèle Auzanneau, Margaret Bento, Stéphane Bonnery, Josiane Boutet, Lucile Cadet, Danièle Cogis, Claudine Garcia-Debanc, Marc Debono Régine Delamotte, JeanFrançois De Pietro, Marie-Laure Elalouf, Valentin Feussi, Laurent Gajo, Emmanuelle Huver, Christian Lagarde, Mylène Lebon-Eyquem, Fabienne Leconte, Gudrun Ledegen, Nadia Maillard, Maira Mamede, Bruno Maurer, Clara Mortamet, Fanny Rink, Valerie Spaëth, Marielle Rispail, Françoise Ropé, Eguzki Urteaga, Cécile Van den Avenne, Daniel Véronique 